\title{
Endostatin promotes the anabolic program of rabbit chondrocyte
}

\author{
Yi FENG, Yi Pin WU, Xu Dong ZHU, Yan Hong ZHANG, Qing Jun MA* \\ Department of Molecule Genetics, Beijing Institute of Biotechnology, 27 Taiping Road, Beijing 100850, China
}

\begin{abstract}
Endostatin is a natural occurred angiogenesis inhibitor derived from collagenXVIII. So far its function during the angiogenesis process of bone formation and arthropathy has not been well studied yet. The present study addresses the function of endostatin in rabbit articular chondrocytes (RAC). We found that endostatin can promote RAC adhesion and spreading as well as its proliferation. In monolayer cultured RAC, CollagenII, TIMP1 and collagenXVIII transcription were up regulated by endostatin while collagenI and MMP9 were down regulated. Moreover collagenXVIII and endostatin antigens are present at synovial fluid. These findings indicate new function of endostatin as a homeostatic factor in cartilage metabolism.
\end{abstract}

Keywords: endostatin, angiogenesis, chondrocyte, matrix metalloproteinase.

\section{INTRODUCTION}

The formation of new capillaries from preexisting blood vessels termed angiogenesis is a key feature in many developmental and pathological processes [1]. The regulation of angiogenesis by pro- and anti-angiogenesis factors is now considered promising for the treatment of angiogenesis disorder disease [2]. Endostatin is a natural angiogenesis inhibitor derived from collagenXVIII NC1 domain. Although it has been used in the clinical test to treat tumor, the mechanism behind still remains unclear $[3,4]$. CollagenXVIII is mainly localized in vascular and epithelial basement membrane, however, it is broadly distributed at organs such as liver, testis, pancreas, brain, lung, skeletal muscle, kidney et al [5-7]. Its presence in avascular tissue has been found in cornea but there were no reports about its detection at cartilage [8]. Chondrocytes, the unique cell type of cartilage, exist in an informationrich extracellular environment consisting of ECM molecules, which could interact with and modulate the

*Correspondence: Qing Jun MA

Tel: +86-10-66931813

E-mail: maqj@vip.sina.com

Abbreviations: rhEN, recombination human endostatin; MMP, matrix metalloproteinase; ECM, extracellular matrix; TIMP, tissue inhibitor of matrix metalloproteinase; RAC, rabbit articular chondrocyte; EC, endothelial cell; HUVEC, human umbilical vein endothelial cell; RA, Rheumatic arthritis; OA, osteoarthritis; CM, conditioned media; $\mathrm{SF}$, synovial fluid. activity of growth factors, hormones and ECM remodeling enzymes. The functional switch of chondrocytes from anabolic program and anti-angiogenesis phenotype toward catabolic program and pro-angiogenesis phenotype is characteristic of chondrocyte aging and pathology [9-11]. The anabolic program is associated with the production of extracellular matrix, protease inhibitors, and cell replication while the catabolic program is related to the secretion of proteases, suppression of matrix synthesis and inhibition of chondrocyte proliferation. ECM is one of the known factors regulating chondrocyte viability and metabolism, but the precise mechanism still above the heads of understanding, and besides, the functional role of ECM proteolytic fragments produced in cartilage degeneration have long been neglected. We newly found that rabbit articular chondrocytes (RACs) express collagenXVIII, and the proteolytic fragment of collagenXVIII namely endostatin (rhEN) could promote RACs adhesion and spreading without inducing the dedifferentiation phenotype. In addition, rhEN stimulates RACs proliferation and induces transcription of TIMP1, collagenII and collagenXVIII while inhibiting MMP9 and collagenI transcription. These findings suggest a homeostatic function of collagenXVIII/endostatin in cartilage metabolism.

\section{MATERIALS AND METHODS}

Probes

Mouse GAPDH, collagenI, collagenII, collagenX probes are kindly gifts from Prof. Xiao YANG. Other Probes were all designed and 
amplified according to each coding sequence from GenBank. Human fetal liver cDNA library (Intvitrogen) or mouse muscle RT-PCR products were used as templates, depending on primers used. The length of each probe and the primers used for amplify were as below: Human TIMP1, Farward: 5'-ATGGCCCCCTTTGAGCC-3'; Reverse: 5'-TCAGGCTATCTGGGACCGC-3'. Mouse MMP2, Farward: 5'-CTCCGGAGATCTGCAAACAG-3', Reverse: 5'CAGCCAGTCTGATTTGATGC-3'; Mouse MMP9, Farward: 5'GCATCTACAGAGTCTTTGAG-3', Reverse: 5'-AGGAGGTCGTAGGTCACGTA-3'; Mouse MMP13, Farward: 5'-TGCTTCCTGATGATGACGTT-3', Reverse: 5'-GCATGACTCTCACAATGCGA-3'; mouse Timp2, Farward: 5'-CGCCTGCAGCTGCTCC3', Reverse: 5'-CGGGTCCTCGATGTCA-3'; Human collagenXVIII, endostatin coding sequence Accession Number: AF416592. All sequences amplified here were cloned into pGEM-Teasy Vector (promega) then confirmed by DNA sequencing.

\section{Production of human endostatin and development of anti- rhEN antibody}

Pichia pastoris expressed human endostatin were purified by heparin-sapherose affinity chromatography as described in our previous works [12]. Human endostatin cDNA was amplified from human fetal liver cDNA library (Clontech Inc.), and subcloned to pPIC9 vector, which was then transformed to GS115 host strain. After cultured for $48 \mathrm{~h}$, the supernatant was collected and the cell free conditioned media was concentrated by ammonium sulfate precipitation (70\%). The precipitated protein was dissolved in $10 \mathrm{mM}$ Tris buffer (pH7.4) containing $150 \mathrm{mM} \mathrm{NaCl}$ and dialyzed overnight at $4^{\circ} \mathrm{C}$. The dialyzed sample was further concentrated by ultrafiltration using MACROSEP ${ }^{\mathrm{TM}}$ centrifugal concentrators (Pall Filtron Corp, MA) and purified at $4^{\circ} \mathrm{C}$ by heparin-sepharose CL6B (Amersham Pharmacia) affinity chromatography. After equilibrated with $10 \mathrm{mM}$ Tris, $150 \mathrm{mM} \mathrm{NaCl}, \mathrm{pH}$ 7.4, the samples were loaded with a flow rate of $20 \mathrm{ml} / \mathrm{h}$ by using a peristaltic pump (Amersham Pharmacia). The column was then washed with equilibration buffer until the A280 was $<0.001$. Bound proteins were eluted by stepwise gradients of $\mathrm{NaCl}(0.3,0.6,1$, and $2 \mathrm{M}$, respectively). Peak fractions from 0.3 to $0.6 \mathrm{M}$ were pooled and dialyzed against PBS ( $\mathrm{pH7}$ 7). The purified protein from yeast expression system was further characterized by $\mathrm{N}$-terminal sequenceing, and the molecule weight of rhEN was determined by MS method. The purity of rhEN was further verified by HPLC and the protein concentration was measured by the BCA assay (Pierce). Recombinant human endostatin expressed from pichia system was used in all in vitro assays in this study. Purified rhEN were stored at $-70^{\circ} \mathrm{C}$ before use. $\mathrm{Balb} / \mathrm{c}$ mice (provided by experimental animal center) was immunized, through sc. injection of $50 \mu \mathrm{g}$ rhEN with Freund's adjuvant two additional boost injections were followed $50 \mu \mathrm{g}$ rhEN with an interval of $7 \mathrm{~d}$. The activity of serum antibodies was determined by ELISA. The immunized mice were then sacrificed and the collected serum was stored at $-20^{\circ} \mathrm{C}$ before use.

\section{Cell culture}

Human umbilical vein endothelial cells (HUVECs) were isolated from human umbilical cord vein by collagease treatment as described [13] and cells was used at passages 3-6. Cells were grown at $37^{\circ} \mathrm{C}$ in IMDM medium (Hyclone) supplemented with $20 \%$ fetal bovine serum (Hyclone), 100 units $/ \mathrm{ml}$ penicillin, $100 \mu \mathrm{g} / \mathrm{ml}$ streptomycin, 2 $\mathrm{ng} / \mathrm{ml}$ bFGF (Bai-Lu-Yuan Biotech, Beijing) and 5 units/ml heparin Sodium (Sigma) in a humidified mixture of $95 \%$ air and $5 \% \mathrm{CO}_{2}$.
Chondrocytes were obtained from the articular cartilage of the knees of young male New Zealand rabbits by sequential enzymatic digestion as previously described [14]. After final digestion, the isolated RACs were resuspended in media containing Ham's-F12/ DMEM (1:1) (Hyclone), 12\% fetal bovine serum and 100 units $/ \mathrm{ml}$ of penicillin and streptomycin. Cells were then incubated in a humidified environment at $37^{\circ} \mathrm{C}$ in presence of $5 \% \mathrm{CO}_{2}$. Cell generations from 1 to 3 were used.

\section{Cell adhesion assay}

rhEN $(100 \mu \mathrm{g} / \mathrm{ml})$ was immobilized on 96-well non-tissue culture-treated plates (Costar) as described previously [15]. The coating efficiency of endostatin was measured by anti-endostatin enzyme-linked immunosorbent assays (data not shown). Wells were washed and incubated with $1 \% \mathrm{BSA}$ in PBS for $1 \mathrm{~h}$ at $37^{\circ} \mathrm{C}$ to block nonspecific cell attachment. Subconfluent RACs were harvested, washed, and resuspended in adhesion buffer containing DMEM medium, $1 \mathrm{mM} \mathrm{MgCl} 2,0.2 \mathrm{mM} \mathrm{MnCl}$, and $0.5 \%$ BSA. Aliquot of $200 \mu \mathrm{l}$ containning $1 \times 10^{5}$ RACs was added and allowed to attach for $30 \mathrm{~min}$ at $37^{\circ} \mathrm{C}$. After washing, the attached cells were stained for 10 min with crystal violet, and cell-associated crystal violet was eluted by addition of $100 \mu 1$ of $10 \%$ acetic acid. Cell adhesion was quantified by measuring the optical density of eluted crystal violet at a wavelength of $570 \mathrm{~nm}$ using a microtiter plate reader (Bio-Rad).

\section{Cell proliferation assay}

The growth stimulating effect of rhEN on RAC was tested by [3H]-thymidine incorporate method as described previously [16]. The cells were plated at $1 \times 10^{5}$ cells/well for HUVECs in 96-well plates and $0.6 \times 10^{5}$ cells/well for RACs. After $24 \mathrm{~h}$ incubation at $37^{\circ} \mathrm{C}$, the medium was replaced by $2 \%$ FBS. For HUVECs, medium contains $5 \mathrm{ng} / \mathrm{ml}$ of bFGF together with or without $1 \mu \mathrm{g} / \mathrm{ml} \mathrm{rhEN}$. For RACs, the medium contains $1 \mu \mathrm{g} / \mathrm{ml} \mathrm{rhEN}$ or not. Then cells were pulsed with $1 \mu \mathrm{Ci}$ of [3H]-thymidine (Yanhui biotech., Beijing) for $4 \mathrm{~h}$. Cells were washed three times with PBS, digested with trypsin, transferred to nitrocellulose membrane, and heat dried at $60^{\circ} \mathrm{C}$ for $1 \mathrm{~h}$. Cell associated radioactivity was determined by a scintillation counter (Please provide the name of the supplier). Cell viability was represented as CPM value.

\section{Cell cycle analysis}

RAC cells were starved for $24 \mathrm{~h}$ in F12/DMEM (1:1) with 1\% FBS. Then the cells were cultured in fresh F12/DMEM (1:1) supplemented with $1 \% \mathrm{FBS}$ with or without $10 \mu \mathrm{g} / \mathrm{ml} \mathrm{rhEN}$ at $37^{\circ} \mathrm{C}$ for 12 $\mathrm{h}$. The cells were harvested, fixed in ethanol at $0^{\circ} \mathrm{C}$ for $10 \mathrm{~min}$ and washed twice in PBS. After addition of $100 \mu 1$ RNase A $(1 \mathrm{mg} / \mathrm{ml})$ and propidium iodide $(925 \mu \mathrm{g} / \mathrm{ml})$, the samples were subjected to a flow cytometer (FACScalibur, BD) For analysis. Results were achieved by using Cell Quest software and ModFit LT2.0.

\section{RNA extraction and Northern blot analysis}

Subconfluent RACs were incubated at serum free media for $24 \mathrm{~h}$, subjected to treatment of $5 \mu \mathrm{g} / \mathrm{ml} \mathrm{rhEN}$ or PBS (control) for $48 \mathrm{~h}$. Total cellular RNAs was extracted by using the TRIZOL Reagent (Life Technologies). Aliquots RNA of $30 \mu \mathrm{g}$ were loaded on each lane, seperated by electrophoresis on $1 \%$ agarose gel and transferred to nylon membranes (Zeta-Probe GT; Bio-Rad). The membranes were hybridized with $\alpha-{ }^{32} \mathrm{P}$-labelled (deoxyCTP) collagen II, CollagenX, CollagenI, CollagenXVIII, MMP9, MMP2, MMp13, TIMP2, TIMP1 and GAPDH probes, respectively [17]. GAPDH 
was used as loading control.

\section{Western blot analysis}

Total cellular proteins were prepared by lysing cells in $20 \mathrm{mM}$ Tris- $\mathrm{HCl}$ (pH 8.0), $150 \mathrm{mM} \mathrm{NaCl}, 2 \mathrm{mM}$ EDTA, 1\% Triton X-100, $5 \mu \mathrm{g} / \mathrm{ml}$ aprotinin, $5 \mu \mathrm{g} / \mathrm{ml}$ leupeptin, $5 \mu \mathrm{g} / \mathrm{ml}$ pepstatin, and $1 \mathrm{mM}$ PMSF. Rabbit joint synovials were aspirate from knee joint and dilute in the same buffer. RACs conditioned medium was used directly. Protein of 30 40 $\mu \mathrm{g}$ was separated by $10 \%$ SDS-PAGE and electrophoretically transferred to nitrocellulose filters. The filters were blocked in 5\% BSA in Tris-buffered saline ( $\mathrm{pH} 7.5)$ containing $0.1 \%$ Tween 20 and then incubated for $2 \mathrm{~h}$ at $37^{\circ} \mathrm{C}$ with anti-rhEN and then the second antibody. BCIP-NBT reagent (Invitrogen) were used to detect the signals.

\section{Statistics}

Each experiment was repeated at least three times. Data were represented as Mean $\pm \mathrm{SE}$ and analyzed using paired student's $t$ test. $P$ values of no more than 0.05 were considered as significant.

\section{RESULTS}

CollagenXVIII expression in RAC and synovial fluids

CollagenXVIII mRNA expression in chondrocytes was detected by Northern blotting. It was found to be present on three different primary isolated RACs, as well as on cultured in vitro between passages 2 3 (Fig. 1A,). Without in vitro stimulation, collagenXVIII mRNA can be detected in all samples as a smeared band of approximately $5.0 \sim 6.0 \mathrm{~kb}$, which does not exist in freshly isolated rabbit muscle RNAs (data not shown). Four bands in mouse and three in human can be detected with consensus sequence probe [5-7]. In this study, C-terminal consensus endostatin segment was used to ensure that each RAC transcription variants could be detected. Limited by knowledge about orthogonal gene of collagenXVIII in rabbit, we failed to show which variant was expressed by RAC.

The collagenXVIII $\alpha 1$ protein synthesized by RACs and presented in synovial fluids were detected by Western blot. The full length glycosylated collagenXVIII $\alpha 1$ chain can be found in all three samples (Fig.1B) as a band larger than $200 \mathrm{kD}$. There is a rich supply SF sample, less in RAC extracts and faint in CF sample. Another band of 58 $\mathrm{kD}$ might represents partially digested C-terminal collagenXVIII $\alpha 1$ chain only be detected in SF sample (Fig. 1B, lane 3).

\section{RhEN stimulate RAC proliferation and drive RAC cell} cycle progression while inhibit HUVEC proliferation

Next we wanted to detect the influence of rhEN on viability of RACs . It was unexpected to found that rhEN could stimulate RAC proliferation. In order to rule out the possible methodological mistakes, the inhibition effect of endostatin on HUVECs was also examined (Fig. 2B, $P<$ $0.05)$. Given that the primary isolated RACs stay resting at $\mathrm{G}_{0}$ phase for a long time in conventional media without
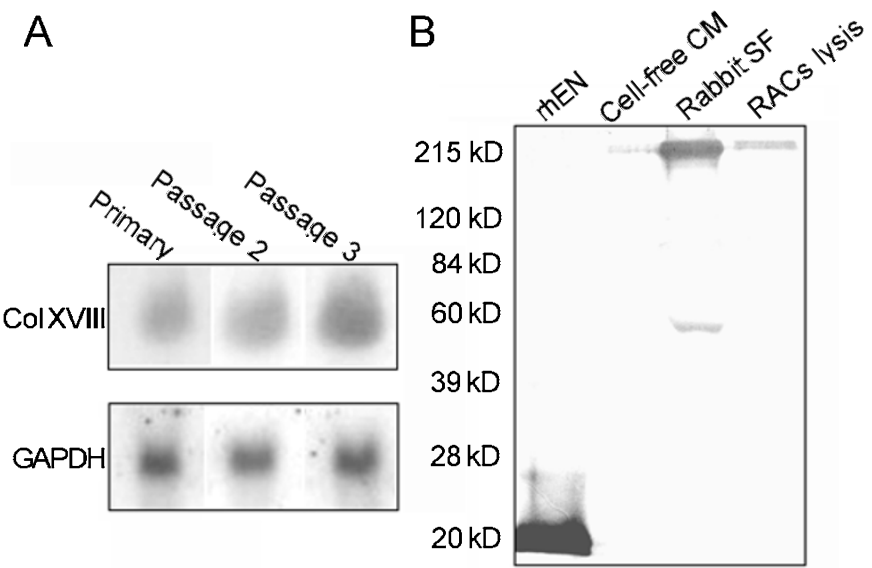

Fig. 1 The expression of CollagenXVIII in joint tissue with chondrocyte as one of its origins. (A) CollagenXVIII expression was detected by Northern blot. Endostatin coding sequence was used as probe, GAPDH was used as an internal control. (B) CollagenXVIII $\alpha 1$ protein synthesized by RACs in synovial fluids were detected by Western blot. Purified rhEN was used as positive control. Molecule weight is indicated as shown here. Three independent tests were performed and one representative result was shown here. with separate sample preparations and the obtained results are consistent as shown here.
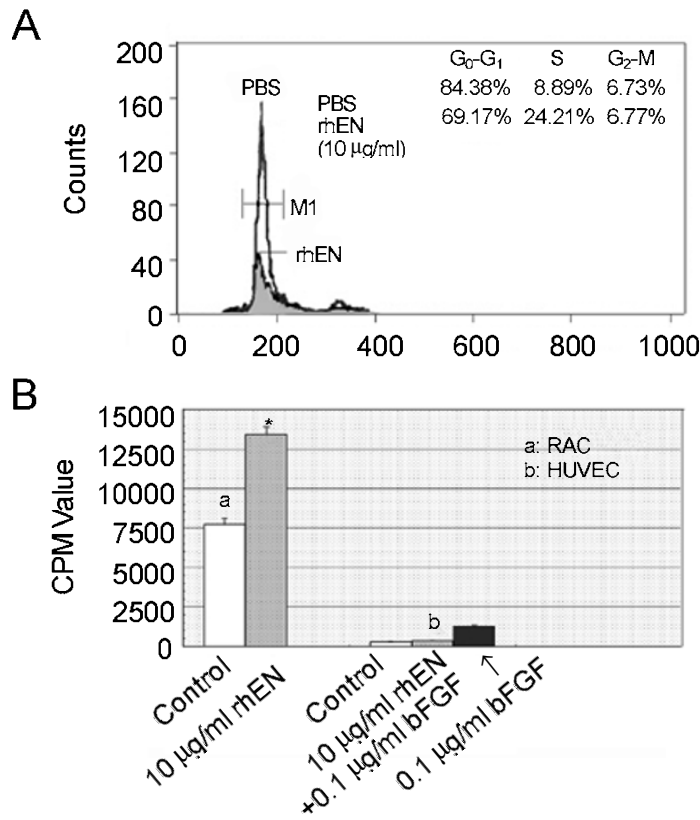

Fig. 2 rhEN promotes RACs cell cycle processing. (A) The cell cycle of RACs was detected by flow cytometry. Cell were treated by $10 \mu \mathrm{g} / \mathrm{ml} \mathrm{rhEN} \mathrm{at} 37^{\circ} \mathrm{C}$ for $12 \mathrm{~h}$ or by PBS, which served as control. (B) The growth stimulating effect of rhEN on RAC was tested by $[3 \mathrm{H}]$-thymidine incorporate method. Both RAC cells (a) and HUVC cells (b) were tested. The experiment were repeated at least three times with conformable results. * represents $P<0.05$. 
any supplements, we presumed that rhEN stimulate RACs proliferation by promoting its cell cycle progression. The FACs data shown here indicates that solely treatment with rhEN could restore DNA synthesize in the resting RACs (Fig. 2A).

\section{RhEN facilitates RAC adhesion and promotes its spreading}

It has been reported that immobilized endostatin promotes endothelial cell adhesion and spreading [19, 20]. We observed similar effects on chondrocytes (Fig. 3). The RACs adhesion on immobilized rhEN was three times higher compared to those on none adhesion substrate (BSA) (Fig. 3B). RACs change to a stretching shape when spreading on rhEN (Fig. 3A, a), similar to those spread on

\section{A}

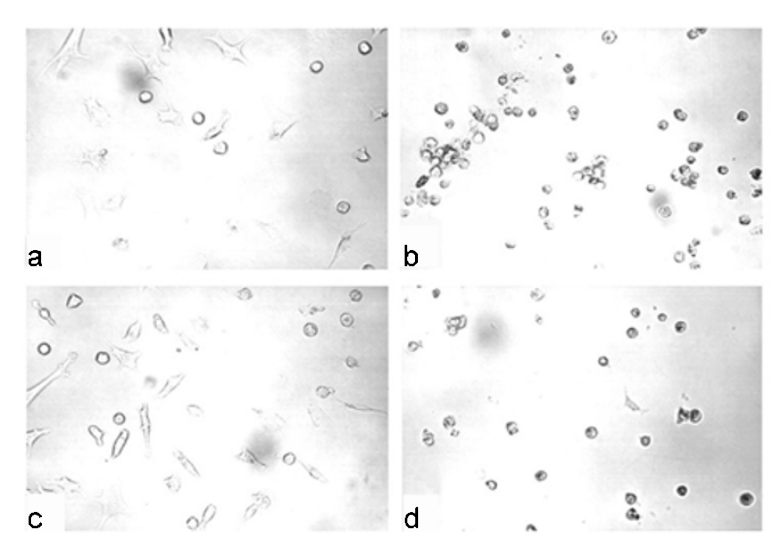

B

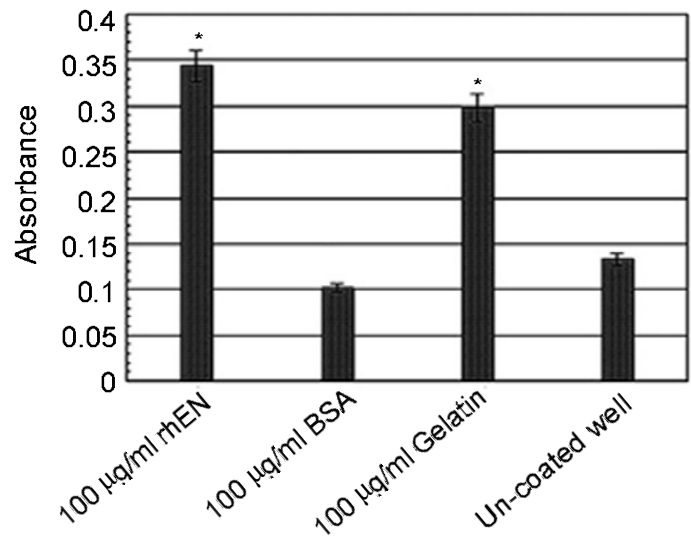

Fig. 3 rhEN support RACs adhering and spreading. RACs prepared as described in "Materials and Methods" were allowed to attach to the 96 well plate, each coated with $100 \mu \mathrm{g} / \mathrm{ml} \mathrm{rhEN}$ (a), $100 \mu \mathrm{g} / \mathrm{ml}$ BSA (b), $100 \mu \mathrm{g} / \mathrm{ml}$ Gelatin (c) or left un-coated (d). (A) RACs morphology when spreading on rhEN. (B) Cell adhesion was quantified by measuring the optical density of eluted crystal violet at a wavelength of $570 \mathrm{~nm} *$ represents $P<0.05$. gelatin (Fig. 3A, c). However, RACs lack of supporting substrates remain in spheral like (Fig. 3B, b). Both the enhanced adhering and spreading effects could be abolished by anti-EN antibody (Fig. 3A, d), further confirming that the enhanced cell spreading and cell adhesion effects were induced by immobilized rhEN.

\section{The expression change of ECM components regulated by rhEN indicate anabolic program}

Because rhEN play a pivotal role in chondrocytes proliferation, it is reasonable to expect the influence of rhEN on RAC differentiation. One of the important character of chondrocyte differentiation was the changes of its biosynthesis ability, so we chose several genes implicated in this process as indicators to serve this purpose. From the results we can see that, transcription of collagen II, which is the typical chondrocyte marker, was up regulated by rhEN in RAC while the chondrocyte dedifferentiation marker collagen I was down regulated (Fig. 4). MMP9, which indicats the catabolic functional program in chondrocytes, was down regulated by rhEN concert with the upregulation of its opponent TIMP1. Collagen X was absent from all samples, indicating very rare if not none hypertrophic chondrocytes existed. Two other tests here namely MMP13 and TIMP2 were found to be unaffected by rhEN. As indicated in Fig. 4, the transcription of collagenXVIII was also up regulated by rhEN. This interesting phenomenon leads to the hypothesis about the protective feed back loop of collagenXVIII/endostatin in cartilage, which will be discussed in detail below.

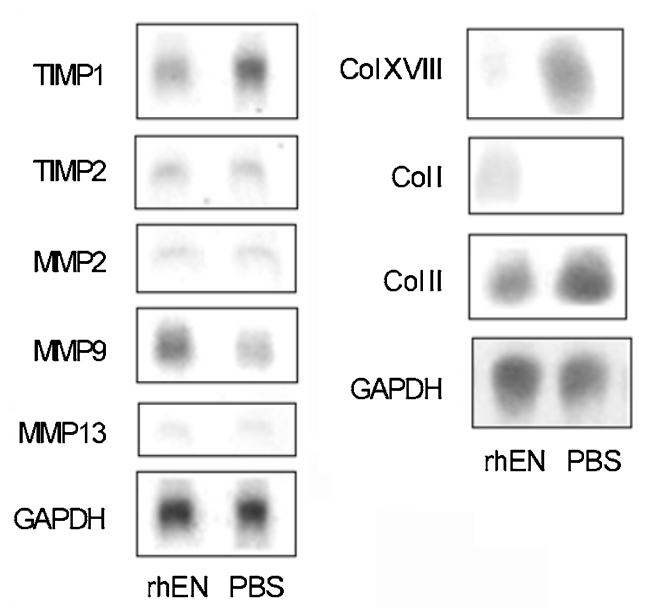

Fig. 4 Expressions of several genes implicated in chondrocyte differentiation were detected by Northern blot. RACs were incubated in serum free media for $24 \mathrm{~h}$, treated reseparately with rhEN $(25 \mu \mathrm{g} / \mathrm{ml})$ or equal volume of PBS ( $\mathrm{pH}$ 7.4) for $48 \mathrm{~h}$. The total cellular RNA was extracted and probed as described in "Materials and methods". 


\section{DISCUSSION}

The present study analyzed the expression and functional properties of collagen XVIII/endostatin in chondrocytes. Collagen type XVIII can be produced by chondrocyte both in vitro and in vivo. In vitro cultured RACs can be regulated by endostatin. The most significant observation herein is the collagenXVIII expression in chondrocytes can be up regulated by endostatin which is the proteolytic product of collagenXVIII. This effect mimics the established auto-stimulating feed back mechanism by which the expression of cytokines was regulated by itself. So far this is the first description of such mechanism in the regulation of collagen gene expression and the biological significance is prominent especially in cartilage.

Un-reversible lose of cartilage is partially caused by excessive proteolysis of cartilage matrix protein which is one of the main aspects of arthropathy such as OA and RA [11]. In accord with the auto-stimulatory mechanism, we presented here the proteolysis of collagenXVIII let free the sequestered endostatin, which in turn enhance the collagenXVIII expression and further increase the endostatin concentration. Subsequently, the free active endostatin exert dual function to shut off the cartilage destruction. One is the well established anti-angiogenesis activity of endostatin that prevent the cartilage from being invaded by excessive growth of vessels. The other is demonstrated in our investigations that endostatin induces chondrocyte anabolic program to restore the cartilage homeostasis.

In our studies we provided evidence to elucidate the role of rhEN in the regulation of cell proliferation and secretory function of RACs. Opposed from its inhibition effect on HUVECs, rhEN could stimulate RACs proliferation in vitro. This phenomenon is not incredible for it is well known that TGF- $\beta$, the prototype mitogenic factor of chondrocytes, has been found to inhibit ECs proliferation [21]. As RACs can adhere and spread on immobilized rhEN, it is most likely that rhEN interacts with an adhesion receptor on the membrane of chondrocytes. Previous studies showed that endostatin mediate endothelial cell adhesion via interacting with integrin $\alpha 5 \beta 1$ and integrin $\alpha 2 \beta 1[19,29,30]$, which is also presented at chondrocytes membrane [22-24]. Similar effects were observed under the treatment of anti- $\beta 1$-integrin antibodies and rhEN, both of which can prevent chondrocyte dedifferentiation to fibroblast-like cells as well as cell death [23]. However, whether integrin $\alpha 5 \beta 1$ is the putative receptor of rhEN on the surface of chondrocytes still remains to be proved.

The potential of rhEN to contribute to the cartilage extracellular matrix metabolism was demonstrated by the induction of mRNAs of TIMP1, collagen II and collagen
XVIII, as well as the inhibition of MMP9 and collagen I. Other MMPs and inhibitors we tested were unaffected by rhEN, indicating that their expressions might be regulated by different mechanisms, which is in consistent with previous knowledge that the expression of MMP9 and MMP2 is controlled by distinguished pathways [25]. The inhibitory effect of rhEN on MMP9 contributes to its anti-angiogenesis function and chondroprotect function as well, because MMP9 has been proved to trigger the angiogenic switch during carcinogenesis [26] and studies on MMP9 knockout mouse showeddelayed hypertrophic chondrocytes apoptosis [27].

The endostatin receptor on the surface of chondrocytes and the signals between rhEN and its downstream gene expressions still remain to be elucidated. Some known receptors of endostatin in ECs such as glypican [28], integrin $\alpha 2 \beta 1 / \alpha 5 \beta 1 / \alpha v \beta 3$ can also be found in chondrocytes $[19,29]$. The present study demonstrates the cartilage homeostatic function of collagenXVIII/endostatin, which represents a protective feed back mechanism, controlling local homeostasis during tissue remolding. This hypothesis is supported by Ergun et al suggesting that endostatin could inhibit angiogenesis by stabilizing newly formed endothelial tube [31]. Our findings provide a new sight to depict full scenes behind endostatin and from which create a new catalogue of tissue-stable-factor.

\section{ACKNOWLEDGEMENTS}

We thank Prof. Yang XIAO for kindly providing the nuclei acid probes. We thank Dr XueYing LIANG for his help in FACscan technique. We thank Dr Hong Feng YUAN for useful advice in HUVECs culture.

Received, Jun 10, 2004

Revised, Dec 10, 2004

Accepted, Dec 22, 2004

\section{REFERENCES}

1 Folkman J. Angiogenesis in cancer, vascular, rheumatoid and other disease. Nat Med 1995; 1:27-31

2 Carmeliet P, Jain RK. Angiogenesis in cancer and other diseases. Nature 2000; 407:249-257.

3 O'Reilly MS, Boehm T, Shing Y, et al. Endostatin: an endogenous inhibitor of angiogenesis and tumor growth. Cell 1997; 88: 277-85.

4 Herbst RS, Lee AT, Tran HT, Abbruzzese JL. Clinical studies of angiogenesis inhibitors: the University of Texas MD Anderson Center Trial of Human Endostatin. Curr Oncol Rep 2001; 3:13140.

5 Muragaki Y, Timmons S, Griffith CM, et al. Mouse Col18a1 is expressed in a tissue-specific manner as three alternative variants and is localized in basement membrane zones. Proc Natl Acad Sci USA 1995; 92:8763-7.

6. Saarela J, Ylikarppa R, Rehn M, Purmonen S, Pihlajaniemi T. 
Complete primary structure of two variant forms of human type XVIII collagen and tissue-specific differences in the expression of the corresponding transcripts. Matrix Biol 1998; 16:319-28.

7 Saarela J, Rehn M, Oikarinen A, Autio-Harmainen H, Pihlajaniemi $\mathrm{T}$. The short and long forms of type XVIII collagen show clear tissue specificities in their expression and location in basement membrane zones in humans. Am J Pathol 1998; 153:611-26.

8 Lin HC, Chang JH, Jain S, et al. Matrilysin cleavage of corneal collagen type XVIII NC1 domain and generation of a $28-\mathrm{kDa}$ fragment. Invest Ophthalmol Vis Sci 2001; 42:2517-24.

9 Gerber HP, Vu TH, Ryan AM, et al. VEGF couples hypertrophic cartilage remodeling, ossification and angiogenesis during endochondral bone formation. Nat Med 1999; 5:623-8.

10 Harper J, Kalgsbrun M. Cartilage to bone - angiogenesis leads the way. Nat Med 1999; 5:617-8.

11 Thomas M Hering. Regulation of chondrocyte gene expression. Front Biosci 1999; 4:d743-61.

12 Feng Y, Cui LB, Liu CX, Ma QJ. Inhibition effect in vitro of purified endostatin expressed in Pichia pastoris. Chinese $\mathrm{J}$ of Biotechnol 2001; 17:278-82. (In Chinese)

13 Feng Y, Liu CX, Ma QJ. The inhibited effect of human endostatin on HUVEC. Lett Biotechnol 2002; 13:138-40. (In Chinese)

$14 \mathrm{Wu}$ YP, Feng Y, Yang X, Huang CF. TGF- $\beta 1$ is required for maintaining cultured articular chondrocytes in vitro. Lett in Biotechnol 2002; 13:148-51. (In Chinese)

15 Brooks PC, Klemke RL, Schon S, et al. Insulin-like growth factor receptor cooperates with integrin alpha $\mathrm{v}$ beta 5 to promote tumor cell dissemination in vivo. J Clin Invest 1997; 99:1390-8.

16 Dhanabal M, Ramchandran R, Volk R, et al. Endostatin: yeast production, mutants, and antitumor effect in renal cell carcinoma. Cancer Res 1999; 59:189-97.

17 Woods VL Jr, Schreck PJ, Gesink DS, et al. Integrin expression by human articular chondrocytes. Arthritis Rheum 1994; 37: 537-44.

18 Halfter W, Dong S, Schurer B, Cole GJ. Collagen XVIII is a basement membrane heparan sulfate proteoglycan. J Biol Chem 1998; 273:25404-12.

19 Rehn M, Veikkola T, Kukk-Valdre E, et al. Interaction of endostatin with integrins implicated in angiogenesis. Proc Natl
Acad Sci U S A 2001; 98:1024-9.

20 Dixelius J, Cross M, Matsumoto T, et al. Endostatin regulates endothelial cell adhesion and cytoskeletal organization. Cancer Res 2002; 62:1944-7.

21 Sankar S, Mahooti-Brooks N, Bensen L, et al. Modulation of transforming growth factor beta receptor levels on microvascular endothelial cells during in vitro angiogenesis. J Clin Invest 1996; 97:1436-46.

22 Lapadula G, Iannone F, Zuccaro C, et al. Integrin expression on chondrocytes: correlations with the degree of cartilage damage in human osteoarthritis. Clin Exp Rheumatol 1997;15:247-54.

23 Attur MG, Dave MN, Clancy RM, et al. Functional genomic analysis in arthritis-affected cartilage: yin-yang regulation of inflammatory mediators by alpha 5 beta 1 and alpha $\mathrm{V}$ beta 3 integrins. J Immunol. 2000;164:2684-91.

24 Durr J, Goodman S, Potocnik A, von der Mark H, von der Mark K. Exp Cell Res 1993; 207:235-44.

25 Bian J, Sun Y. Transcriptional activation by p53 of the human type IV collagenase (gelatinase A or matrix metalloproteinase 2) promoter. Mol Cell Biol 1997; 17:6330-8

26 Bergers G, Brekken R, McMahon G, et al. Nature Cell Biol 2000; 2:737-44.

$27 \mathrm{Vu}$ TH, Shipley JM, Bergers G, et al. MMP-9/gelatinase B is a key regulator of growth plate angiogenesis and apoptosis of hypertrophic chondrocytes. Cell 1998; 93:411-22.

28 Karumanchi SA, Jha V, Ramchandran R, et al. Cell surface glypicans are low-affinity endostatin receptors. Mol Cell 2001; 7:811-22.

29 Furumatsu T, Yamaguchi N, Nishida K, et al. Endostatin inhibits adhesion of endothelial cells to collagen I via $\alpha 2 \beta 1$ integrin, a possible cause of prevention of chondrosarcoma growth. J Biochem 2002; 131:619-26.

30 Sudhakar A, Sugimoto H, Yang C, et al. Human tumstatin and human endostatin exhibit distinct antiangiogenic activities mediated by $\alpha v \beta 3$ and $\alpha 5 \beta 1$ integrins. Proc Natl Acad Sci USA 2003; 100:4766-71.

31 Ergun S, Kilic N, Wurmbach JH, et al. Endostatin inhibits angiogenesis by stabilization of newly formed endothelial tubes. Angiogenesis 2001; 4:193-206. 